\title{
Heavy drugs: an emerging tool for an improved half- life of the drugs and lead compounds
}

\section{Editorial}

The deuterium switching for hydrogen is an emerging trend for the past decade to improve the bioavailability of the lead compounds and drugs. Since 1970, there are totally 412 patents (obtained by SciFinder search) have been registered with the deuterated analogs and out of them 319 patents have been filed within the past seven years (from 2010, 75\%), implicated the paramount importance of the deuteration of drugs.

The drug has to experience two important processes such as pharmacokinetics and pharmacodynamics when it enters in to the body. In pharmacodynamics, the drug molecules have to interact with the target receptor to create the desired therapeutic effect, and this interaction is directly correlated to the drug concentration. The pharmacokinetic process involves adsorption, distribution, metabolism, excretion, and toxicity (ADMET). If the molecules are metabolized and excreted very quickly during the pharmacokinetics, then they are no longer available to produce the desired therapeutic effect independent of efficacy. To slow down the metabolic process and improve the half-life of the lead compounds/drugs scientist have been using a strategy -incorporation of deuterium in the drug candidates- heavy drugs (deuterated drugs). The improved half-life which in turn lower the dose of the medication and limit the formation of toxic metabolites in some cases. ${ }^{1-5}$

Theory behind the deuteration strategy: The size of a molecule is indistinguishable when the hydrogen atom is replaced in a drug candidate/ lead molecule by its non-radioactive isotope deuterium (D). But their physical and chemical properties entirely rely on their exchange position. As we all know, C-D bond has a very low zeropoint energy and 6-10 fold stronger than the $\mathrm{C}-\mathrm{H}$ bond, which gives a tough task to the metabolizing enzymes to break the C-D bond during the phase I metabolizing process. The change in the rate of bond breaking results in the primary kinetic isotopic effect $\left(1^{0} \mathrm{KIE}\right)$ and slows down the metabolic pathway. It leads to the reduced systemic clearance and resulting in an improved half-life and bioavailability of the deuterated molecule compare to its parent. ${ }^{6-8}$

There are few companies have been working on deuterated drugs and analogs. E.g, Concert Pharmaceuticals, Inc, Auspex Pharmaceuticals, Teva Pharmaceuticals, Pfizer and DeuteRx. Recently reported that the success of CTP-656 (deuterated analog of ivacaftor), a cystic fibrosis transmembrane conductance regulator (CFTR) potentiator. Its deuterated analog has an improved half-life (15h) compare to the parent (12h) molecule. Since they block the key sites involved in the metabolism, the pharmacokinetic properties have been altered and supported the concept of deuteration. ${ }^{9}$ However, the deuterated version can have the same or enhanced half-life, but that completely relies on the structure of the molecule and the metabolic pathways. ${ }^{10}$

The following points have to be considered when you change hydrogen to deuterium ${ }^{1-4}$

i. Is it really going to give the difference in biological properties?
Volume I Issue I - 2017

\author{
Michael Rajesh Stephen \\ Department of Chemistry \& Biochemistry, University of \\ Wisconsin, USA
}

Correspondence: Michael Rajesh Stephen, Department of Chemistry \& Biochemistry, University of Wisconsin, Milwaukee, 3210 North Cramer Street, Milwaukee,Wisconsin 5321 I, USA, Tel + 4 14-7I2-4074, Email stephenm@uwm.edu

Received: March 23, 2017| Published: March 31, 2017

ii. Not only metabolism, sometimes the deuteration can prevent or slow down the epimerization or the interconversion of certain enantiomers (when you exchange at the chiral center).

iii. For an improved half-life, C-D bond breaking should be the key step in the metabolic process. If an alternative metabolic pathway is predominant, then there won't be an improved halflife and the deuteration strategy may not be positive.

iv. To get a patent for a reported molecule: It is obvious to improve the pharmacokinetic profile of a drug or lead molecule by replacing deuterium with the hydrogen, hence unexpected results should be achieved by deuteration. Otherwise, the patent officer will raise an objection (obviousness) for the claim/composition of matter.

\section{Conclusion}

To conclude, deuteration strategy is not going to complement for all cases, but there will be situations where you can improve the function of the compounds.

\section{Acknowledgements}

None.

\section{Conflict of interest}

The author declares no conflict of interest.

\section{References}

1. EJ Velthuisen, TM Baughman, BA Johns, et al. Synthesis and pharmacokinetic profile of highly deuterated brecanavir analogs. Eur J Med Chem. 2013;63:202-212.

2. Wolen RL. The Application of Stable Isotopes to Studies of Drug Bioavailability and Bioequivalence. J Clin Pharmacol. 1986;26(6):419-424.

3. KC Buteau (2009) Deuterated Drugs: Unexpectedly nonobvious. J High Tech L. 2009;22(2009):2-53.

4. GS Timmins. Deuterated drugs: where are we now. Expert Opin Ther Pat. 2014;24(10):1067-1075.

5. AJ Morgan. Deuterated CFTR potentiators. 2014. US 8865902 B2.

6. A Mullard. Deuterated drugs draw heavier backing. Nat Rev Drug Discov. 2016;15(4):219-221. 
7. R Sharma, TJ Strelevitz, H Gao, et al. Deuterium Isotope Effects on Drug Pharmacokinetics. I. System Dependent Effects of Specific Deuteration with Aldehyde Oxidase Cleared Drugs. Drug Metab Dispos. 2012;40(3):625-634.

8. B Halford. Deuterium switcheroo breathes life into old drugs. Chem Eng News. 2016;94(27):32-36.
9. A M Thayer. Biocatalysis. Chem Eng News. 2017;90(22):pp.13-18.

10. R Jahan, MR Stephen, GS Forkuo, et al. Optimization of substituted imidazobenzodiazepines as novel asthma treatments. Eur J Med Chem. $2017 ; 126: 550-560$ 